\section{Evidências do impacto da suplementação de vitamina A no grupo materno-infantil}

\author{
Evidence of the impact of vitamin A \\ supplementation on maternal and child health
}

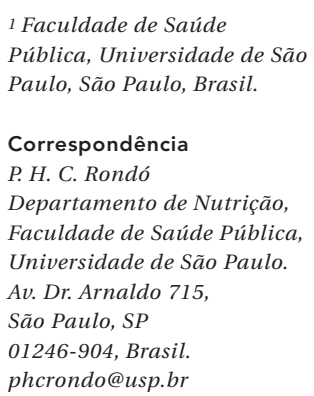

\begin{abstract}
The aim of this article was to collect the results of systematic reviews and meta-analyses that evaluated the effect of vitamin A supplementation on child growth and maternal, fetal, and child morbidity and mortality. A detailed search was performed in PubMed, Cochrane Library, LILACS, PAHO, CAPES, USP Digital Thesis Library, and UNIFESP Collection Database. A total of 14 studies published from 1993 to 2006 were included in the review. There is evidence that vitamin A supplementation in children is associated with a reduction of $23 \%$ to $30 \%$ in mortality risk and attenuation in the severity of measles and diarrhea. There is no evidence of the intervention's impact on pneumonia incidence or mortality in children without measles. Vitamin A also appears to be protective in children and pregnant women with HIVIAIDS, with a positive effect on child morbidity and mortality and birth weight. There is no evidence that supplementation in pregnant and lactating women reduces infant morbidity and mortality, but there is an indication that vitamin A protects against maternal morbidity.
\end{abstract}

Vitamin A; Vitamin A Deficiency; Maternal and Child Health; Meta-Analysis [Publication Type]
Julicristie Machado de Oliveira 1

Patrícia Helen de Carvalho Rondó 1

\section{Introdução}

A vitamina A é essencial para a proliferação e diferenciação celular, tendo impacto no crescimento, desenvolvimento e imunidade 1.

De acordo com West Jr. et al. 2, estima-se que a deficiência de vitamina A e a xeroftalmia afetem, respectivamente, 127 milhões e 4,4 milhões de crianças pré-escolares em todo o mundo, e que cerca de 7,2 milhões e 6 milhões de gestantes apresentem, respectivamente, deficiência de vitamina A e cegueira noturna.

Green \& Mellanby 3 foram os primeiros a sugerir, com base em estudos em animais, que a vitamina A tem uma ação antiinfecciosa. Evidências clínicas provaram, posteriormente, que a administração de óleo de fígado de bacalhau reduzia em $58 \%$ a mortalidade em crianças hospitalizadas ${ }^{4}$. Sommer et al. 5 notaram uma associação entre deficiência de vitamina A e morbidade e mortalidade por doenças infecciosas, e, a partir de 1990, surgiram os estudos intervencionais de suplementação de vitamina A. Aparentemente as infecções mais fortemente associadas com a deficiência da vitamina são aquelas nas quais a função do epitélio está comprometida, como o sarampo, a diarréia e as doenças respiratórias.

Atualmente recomenda-se a suplementação de rotina de vitamina A durante a infância ${ }^{6}$, gravidez ou em qualquer período da lactação, em áreas nas quais a deficiência de vitamina A é en- 
dêmica 7, especialmente onde a prevalência de HIV/AIDS é elevada ${ }^{8}$.

O objetivo deste artigo é reunir os resultados de revisões sistemáticas e metanálises que avaliam o efeito da suplementação de vitamina A no crescimento, bem como na morbidade (infecções respiratórias, diarréia, sarampo, HIV/AIDS) e mortalidade infantil. Serão reunidos, igualmente, resultados que avaliam esse efeito na morbidade (HIV/AIDS, complicações no parto e puerpério, malformações congênitas) e mortalidade materna e fetal.

\section{Métodos}

Foi realizada uma busca criteriosa por estudos de revisão sistemática ou metanálise que avaliaram o impacto da suplementação de vitamina A em gestantes, lactantes e crianças em idade préescolar. Consideraram-se elegíveis estudos que analisaram como desfechos: mortalidade, morbidade e crescimento e desenvolvimento físico.

As buscas foram realizadas na base de dados bibliográficos PubMed, utilizando-se as seguintes estratégias de busca: "vitamin A"[ti] AND (intervention[ti] OR supplement[ti] OR supplements[ti] OR supplementation[ti]) e "vitamin A"[ti] AND meta-analysis[ti]. A base de dados de revisões sistemáticas da Biblioteca Cochrane também foi pesquisada, com a utilização das seguintes palavras-chave: micronutrient and supplementation:ti e vitamin and supplementation: $t i$. Para a consulta na base EMBASE, empregouse a seguinte estratégia: 'vitamin a'ti AND supplement':ti AND 'review':ab e 'vitamin a':ti AND supplement*:ti AND 'meta analysis':ti. Na base LILACS e no acervo da Organização Pan-Americana da Saúde (OPAS), a estratégia foi a seguinte: "Vitamina A"[Descritor de assunto] and "revisão sistemática"[Palavras] or metanálise[Palavras]. Também foi realizada pesquisa na Biblioteca Digital de Teses e Dissertações da Universidade de São Paulo (USP), no Banco de Teses da Coordenação de Aperfeiçoamento de Pessoal de Nível Superior (CAPES) e no acervo da Biblioteca Central da Universidade Federal de São Paulo (UNIFESP), com utilização dos descritores: "vitamina A", "revisão sistemática" e "metanálise".

\section{Resultados}

Foram localizados 14 estudos de revisão sistemática ou metanálise, publicados entre 1993 e 2006, sobre o impacto da suplementação de vitamina A nos desfechos mortalidade, morbidade e crescimento e desenvolvimento físico
(Tabela 1). Estudos de van den Broek et al. 9 e Oliveira 10 investigaram o impacto da intervenção em gestantes e lactantes, respectivamente. Segundo os autores, a suplementação em doses semanais na gravidez e lactação, utilizada no estudo do Nepal, esteve associada à redução da mortalidade materna por todas as causas (durante a gravidez até a 12a semana pós-parto), da cegueira noturna 11 e da morbidade materna (febre, evacuações aquosas e fraqueza) 12. Contudo, não houve impacto na mortalidade materna por causas obstétricas ou infecciosas 11, na perda fetal, na mortalidade até o sexto mês de vida e na antropometria neonatal 13. Em relação ao efeito, na anemia, da suplementação de vitamina $A$, não houve consenso entre os estudos 14,15,16. Segundo Oliveira 10, os estudos que utilizaram suplementação em megadoses simultâneas para puérperas e lactentes não descrevem efeito protetor na mortalidade 17,18 e na ocorrência e duração de episódios de morbidade infantil 17,19, como diarréia, infecção respiratória e pneumonia.

Revisaram-se três metanálises sobre o impacto da suplementação de vitamina A na mortalidade de crianças. Os resultados das análises agregadas foram semelhantes, como pode ser observado na Tabela 1. Fawzi et al. 20, Glasziou \& Mackerras 21 e Beaton et al. 22 descrevem efeito benéfico da intervenção para a mortalidade geral, por diarréia e por sarampo. Em relação à redução da morbidade, Glasziou \& Mackerras 21 relatam que não há consenso entre os estudos, e Beaton et al. 22 descrevem que não há efeito protetor da suplementação na freqüência, duração e prevalência de diarréia e doenças respiratórias; há somente atenuação da gravidade destas.

Huining et al. ${ }^{23}$, em revisão sistemática com metanálise, investigaram o impacto da suplementação de vitamina A no tratamento de sarampo. Há relato de redução na mortalidade geral e na específica por pneumonia apenas quando são utilizadas duas megadoses da vitamina em crianças com até dois anos, ou quando o suplemento apresenta-se na forma de solução aquosa (emulsão) e não à base de óleo.

Nos estudos de metanálise conduzidos pelo Vitamin A and Pneumonia Working Group $24 \mathrm{e}$ por Ni et al. ${ }^{25}$, não são descritos efeitos benéficos da suplementação em relação à incidência de pneumonia 24 , mortalidade por esta doença 24,25 e duração da internação 25. À semelhança dos resultados descritos por Glasziou \& Mackerras 21, Fawzi et al. 20 e Beaton el al. 22, os resultados da metanálise do Vitamin A and Pneumonia Working Group 24 são compatíveis com efeito protetor para a mortalidade geral no grupo de 6 a 11 meses. 
Revisões sistemáticas e metanálises sobre o impacto da suplementação de vitamina $A$.

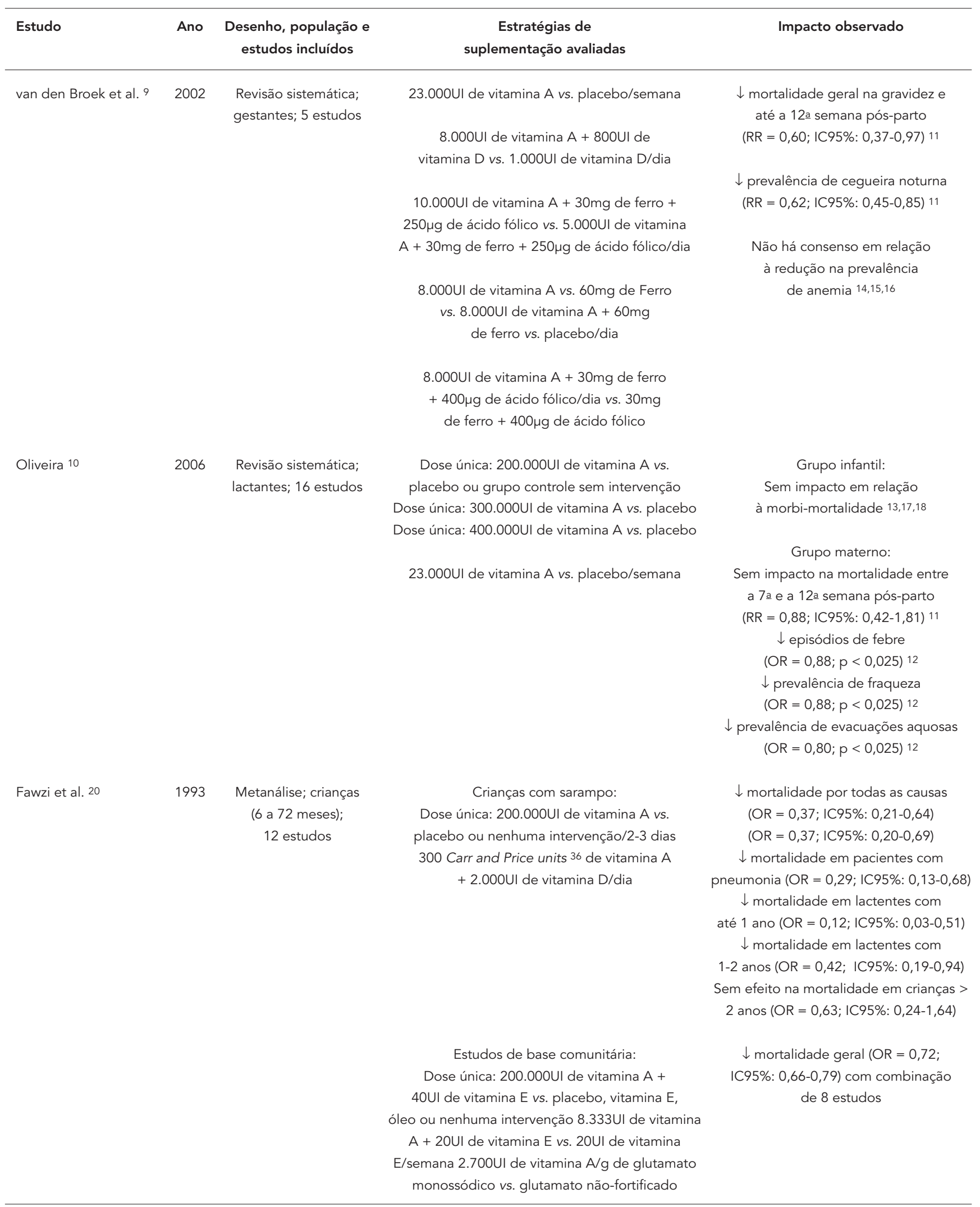

(continua) 
Tabela 1 (continuação)

\begin{tabular}{|c|c|c|c|c|}
\hline Estudo & Ano & $\begin{array}{l}\text { Desenho, população e } \\
\text { estudos incluídos }\end{array}$ & $\begin{array}{c}\text { Estratégias de } \\
\text { suplementação avaliadas }\end{array}$ & Impacto observado \\
\hline & & & & $\begin{array}{c}\downarrow \text { mortalidade geral (OR =0,74; } \\
\text { IC95\%: 0,67-0,81) com combinação } \\
\text { de } 7 \text { estudos } \\
\downarrow \text { mortalidade geral (OR =0,76; } \\
\text { IC95\%: 0,68-0,84) com combinação } \\
\text { de } 6 \text { estudos } \\
\downarrow \text { mortalidade por diarréia } \\
\text { (OR =0,69; IC95\%: 0,57-0,84) } \\
\text { Sem efeito na mortalidade por } \\
\text { pneumonia (OR =0,96; IC95\%: } \\
\quad 0,65-1,42) \\
\text { Sem efeito na mortalidade por sarampo } \\
\text { (OR = 0,61; IC95\%: 0,32-1,15) }\end{array}$ \\
\hline Glasziou \& Mackerras 21 & 1993 & $\begin{array}{l}\text { Metanálise; } \\
\text { crianças e adolescentes } \\
\text { (com até } 13 \text { anos); } \\
12 \text { estudos }\end{array}$ & $\begin{array}{c}\text { Estudos de base comunitária: } \\
\text { 200.000Ul de vitamina A } \\
\text { semestralmente ou quadrimestralmente } \\
\text { Dose única: } 200.000 U 1 \text { de vitamina A } \\
\text { 8.333UI de vitamina A/semana }\end{array}$ & $\begin{array}{c}\downarrow \text { mortalidade por todas as causas } \\
\text { (OR = 0,70; IC95\%: 0,62-0,79) } \\
\downarrow 39 \% \text { das mortes por diarréia } \\
\text { (IC95\%: } 24-50) \\
\downarrow 34 \% \text { das mortes por outras } \\
\text { causas (IC95\%: } 15-49 \text { ) }\end{array}$ \\
\hline & & & $\begin{array}{l}\text { Recém-nascidos com muito baixo peso: } \\
2.000 \mathrm{Ul} \text { a } 4.000 \mathrm{Ul} \text { de vitamina } \mathrm{A} / 2 \text { dias }\end{array}$ & $\begin{array}{c}\downarrow \text { mortalidade por todas as causas } \\
\text { (OR =0,34; IC95\%: 0,15-0,77) } \\
\downarrow 70 \% \text { das mortes por causas respiratórias } \\
\text { (IC95\%: 15-90; } p=0,02) \\
\text { Não há consenso em relação à redução } \\
\text { da displasia bronco-pulmonar e } \\
\text { da ventilação mecânica }\end{array}$ \\
\hline Beaton et al. 22 & 1994 & $\begin{array}{c}\text { Metanálise; } \\
\text { crianças } \\
\text { (6 meses a } 5 \text { anos); } \\
10 \text { estudos sobre } \\
\text { mortalidade, } \\
23 \text { estudos sobre } \\
\text { morbidade (incluindo os } \\
10 \text { sobre mortalidade) }\end{array}$ & $\begin{array}{l}\text { Administração de altas doses, doses } \\
\text { semanais e utilização de glutamato } \\
\text { monossódico fortificado }\end{array}$ & $\begin{array}{c}\downarrow \text { em } 23 \% \text { na mortalidade por todas as } \\
\text { causas em crianças com idade entre } 6 \\
\text { meses e } 5 \text { anos (RR =0,77; IC95\%: } \\
\qquad 0,68-0,88) \\
\downarrow \text { mortalidade por diarréia } \\
\text { (RR = 0,71; IC95\%: 0,57-0,88; } p=0,002) \\
\downarrow \text { mortalidade por sarampo } \\
\text { (RR = 0,46; IC95\%: 022-0,98; } p=0,043 \text { ) } \\
\text { Sem impacto na mortalidade por } \\
\text { doenças respiratórias (RR }=0,94 ; \\
\text { IC95\%: } 0,63-1,42 ; p=0,777 \text { ) } \\
\text { A suplementação não afetou a freqüência, } \\
\text { duração e prevalência de diarréia e } \\
\text { doenças respiratórias }\end{array}$ \\
\hline $\begin{array}{l}\text { Vitamin A and } \\
\text { Pneumonia Working } \\
\text { Group } 24\end{array}$ & 1995 & $\begin{array}{l}\text { Metanálise; crianças de } \\
\text { países em desenvol- } \\
\text { vimento; } 12 \text { estudos }\end{array}$ & $\begin{array}{l}\text { Baixas doses semanais ou altas doses } \\
\text { semestrais ou uma única administração }\end{array}$ & $\begin{array}{l}\text { Sem impacto na incidência de pneumonia } \\
\text { (RR =0,95; IC95\%: 0,89-1,01) } \\
\text { Sem impacto na incidência de pneumonia } \\
\text { (RR = 0,99; IC95\%: 0,96-1,06) com a } \\
\text { exclusão de um estudo }\end{array}$ \\
\hline
\end{tabular}

(continua) 
Tabela 1 (continuação)

\begin{tabular}{|c|c|c|c|c|}
\hline Estudo & Ano & $\begin{array}{l}\text { Desenho, população e } \\
\text { estudos incluídos }\end{array}$ & $\begin{array}{c}\text { Estratégias de } \\
\text { suplementação avaliadas }\end{array}$ & Impacto observado \\
\hline & & & & $\begin{array}{c}\text { Sem impacto na mortalidade por pneu- } \\
\text { monia (RR = 0,98; IC95\%: 0,75-1,28) } \\
\text { Sem impacto na mortalidade por } \\
\text { pneumonia segundo grupo etário } \\
\text { Mortalidade por todas as causas } \\
\text { em lactentes com até } 1 \text { ano: } \\
\downarrow \text { mortalidade no grupo de } 6-11 \text { meses } \\
\text { (RR = 0,69; IC95\%: 0,54-0,90) } \\
\text { Sem impacto na mortalidade no grupo de } \\
0-5 \text { meses (RR =0,97; IC95\%: } 0,73-1,29 \text { ) }\end{array}$ \\
\hline Grotto et al. 26 & 2003 & $\begin{array}{l}\text { Metanálise; } \\
\text { crianças sem doenças } \\
\text { no baseline ( } 6 \text { meses a } \\
7 \text { anos); } 9 \text { estudos }\end{array}$ & $\begin{array}{c}\text { Dose única: } 200.000 U \text { l de vitamina } A \\
2,3,4 \text { ou } 6 \text { doses de } 200.000 U \text { de vitamina } A \\
10.000 \text { Ul de vitamina } A / \text { semana } \\
\text { por } 40 \text { semanas }\end{array}$ & $\begin{array}{l}\text { Sem impacto na incidência de diarréia } \\
\text { (RR }=1,00 ; \text { IC95\%: 0,94-1,07) } \\
\uparrow \text { incidência de doença respiratória } \\
\text { (RR }=1,08 ; \text { IC95\%: } 1,05-1,11)\end{array}$ \\
\hline Brown \& Roberts 27 & 2004 & $\begin{array}{l}\text { Metanálise; crianças } \\
\text { sem doenças ( } 6 \text { a } 72 \\
\text { meses); } 5 \text { estudos }\end{array}$ & $\begin{array}{l}\text { 100.000, } 200.000 \text { Ul ou } 400.000 \text { Ul de } \\
\text { vitamina A em dose única ou por } \\
2 \text { dias consecutivos }\end{array}$ & $\begin{array}{l}\text { Sem efeito na mortalidade por infecção } \\
\text { respiratória aguda } \\
\qquad(\mathrm{OR}=1,16 ; \text { IC95\%: 0,61-2,21) } \\
\text { Sem impacto no tempo de normalização } \\
\text { da febre, saturação de oxigênio, taxa } \\
\text { respiratória e duração de internação }\end{array}$ \\
\hline Huiming et al. 23 & 2005 & $\begin{array}{l}\text { Revisão sistemática } \\
\text { e metanálise; crianças e } \\
\text { adolescentes com sarampo } \\
\text { (até } 15 \text { anos); } 7 \text { estudos }\end{array}$ & 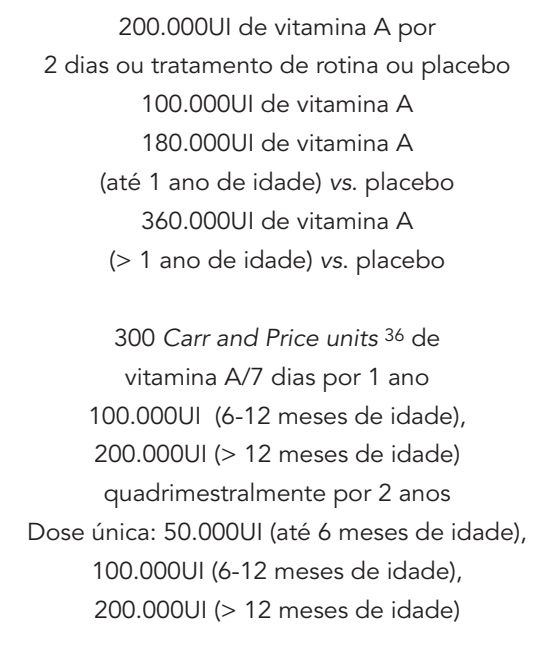 & $\begin{array}{c}\text { Sem impacto da redução da mortalidade } \\
\text { (RR = 0,70; IC95\%: 0,42-1,15) } \\
\text { Utilização de ao menos duas doses } \\
\text { de 200.000UI de vitamina A: } \\
\downarrow \text { mortalidade } \\
\text { (RR = 0,36; IC95\%: 0,13-0,82) } \\
\downarrow \text { mortalidade no grupo com até } 24 \text { meses } \\
\text { (RR }=0,17 \text {; IC95\%: 0,03-0,61) } \\
\downarrow \text { mortalidade específica por pneumonia } \\
\text { (RR }=0,33 \text {; IC95\%: 0,08-0,92) } \\
\text { Sem impacto na mortalidade em crianças } \\
\text { ou adolescentes com mais de } 2 \text { anos } \\
\text { (RR }=0,94 ; \text { IC95\%: 0,23-3,10) } \\
\text { Suplemento em forma de emulsão: } \\
\downarrow \text { mortalidade (RR =0,19; IC95\%: 0,02-0,85) }\end{array}$ \\
\hline $\mathrm{Ni}$ et al. 25 & 2005 & $\begin{array}{l}\text { Revisão sistemática e } \\
\text { metanálise; crianças e } \\
\text { adolescentes sem sarampo } \\
\text { (até } 15 \text { anos); } 5 \text { estudos }\end{array}$ & $\begin{array}{c}\text { 100.000UI de vitamina A (até } 1 \text { ano de idade) } \\
200.000 \text { Ul de vitamina A ( }>1 \text { ano de idade) } \\
150.000 \text { Ul de vitamina A } \\
\text { (100.00 na admissão e } 50.000 \text { Ul no } \\
\text { segundo dia de internação; até } 1 \text { ano de idade) } \\
\text { 300.000Ul de vitamina A } \\
\text { (200.00 na admissão e } 100.000 \text { U no segundo } \\
\text { dia de internação; até } 1 \text { ano de idade) }\end{array}$ & $\begin{array}{l}\text { Sem impacto da redução da } \\
\text { mortalidade associada à pneumonia } \\
(\mathrm{OR}=1,49 ; \text { IC95\%: 0,66-3,35) } \\
\text { Sem impacto na duração da } \\
\text { internação hospitalar } \\
\text { (DMS = 0,08; IC95\%: -0,43-0,59) }\end{array}$ \\
\hline & & & $\begin{array}{l}20.000 \mathrm{UI} / \mathrm{dia} \text { por } 6 \text { dias }+1.500 \mathrm{UI} / \mathrm{dia} \\
\text { por } 20 \text { dias vs. placebo }\end{array}$ & $\begin{array}{c}\downarrow \text { na taxa de recorrência de broncopneu- } \\
\text { monia }(\mathrm{OR}=0,12 ; \text { IC95\%:0,03-0,46) }\end{array}$ \\
\hline
\end{tabular}

(continua) 
Tabela 1 (continuação)

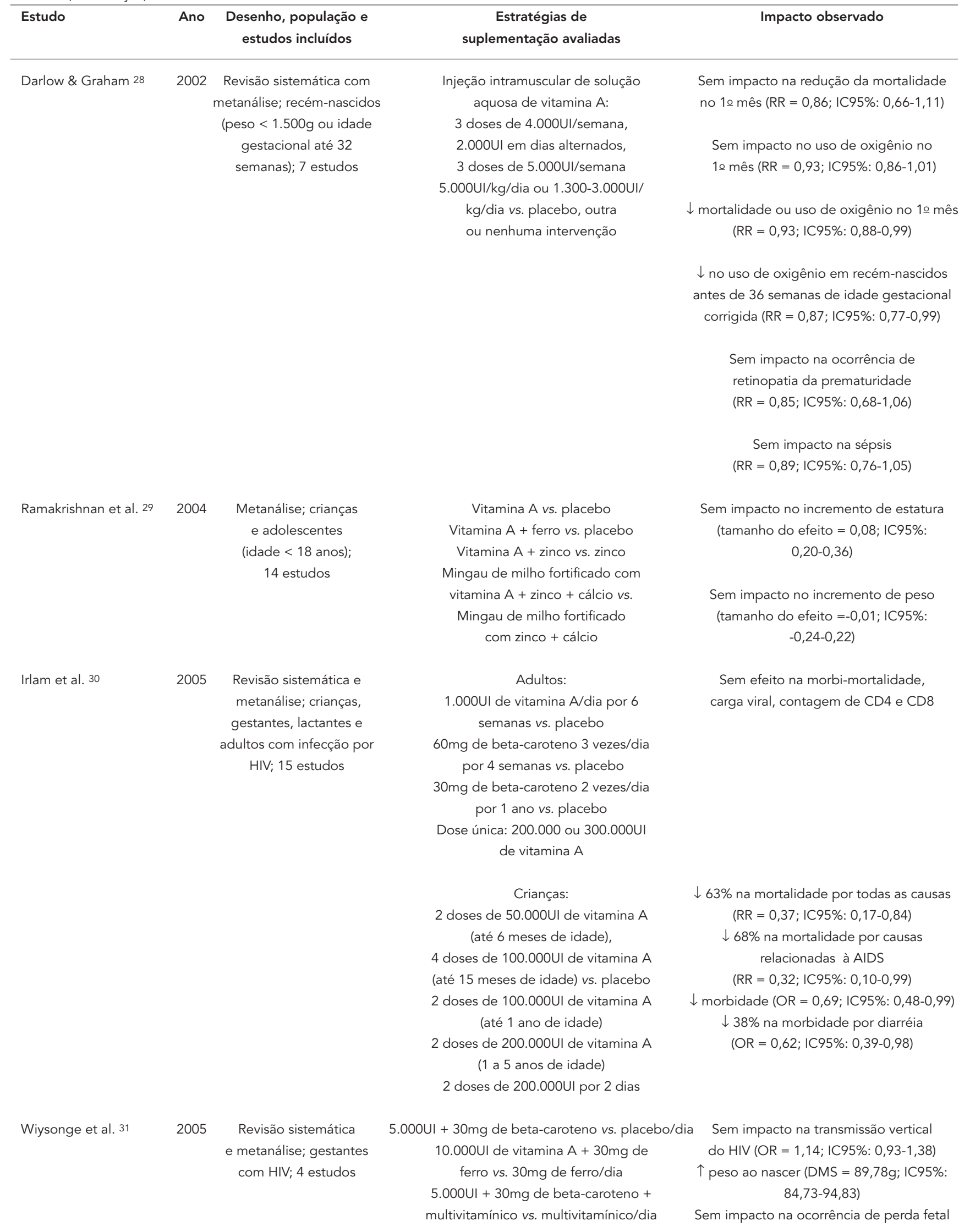

(continua) 


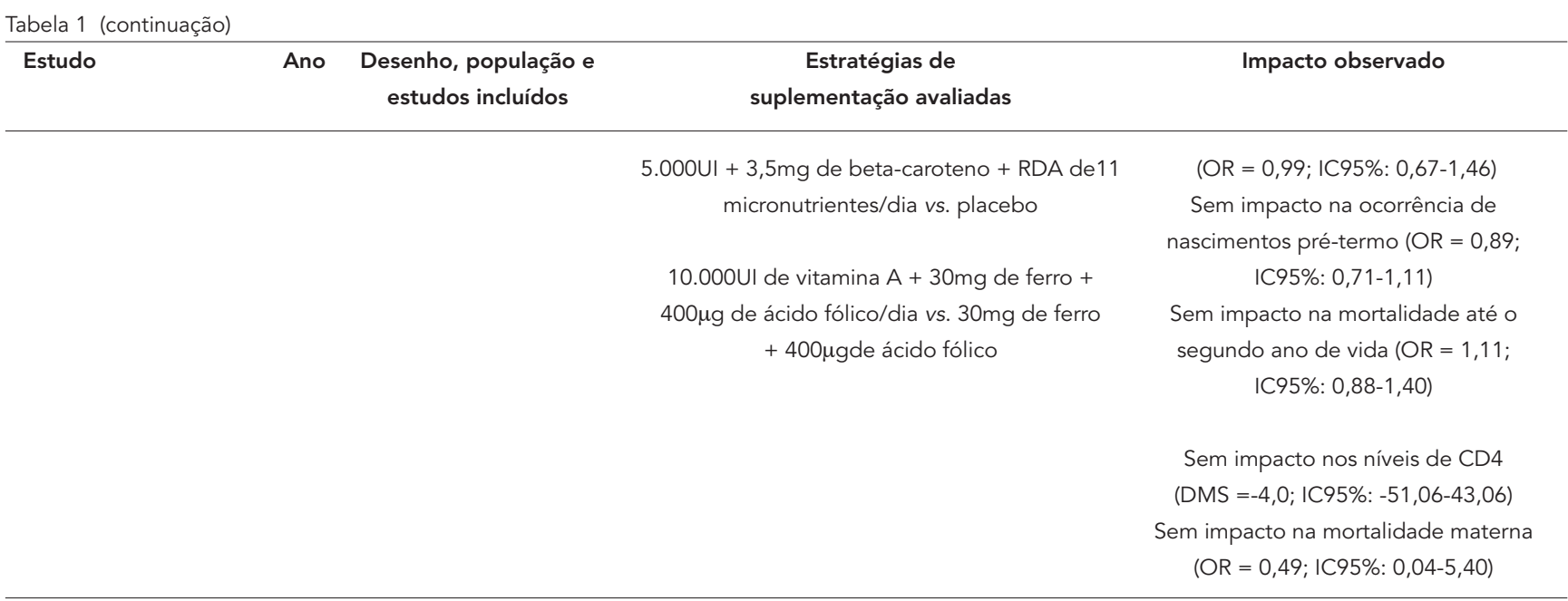

RR: risco relativo; IC95\%: intervalo de confiança de 95\%; OR: odds ratio; DMS: diferença de médias sumária; RDA: recommended dietary allowances.

Nas metanálises subseqüentes conduzidas por Grotto et al. 26 e Brown \& Roberts 27, foram excluídos os resultados de investigações com crianças que apresentaram alguma doença no início do estudo 26 e sinais de deficiência de vitamina A, desnutrição grave, doença grave ou sarampo 27. Os autores não descrevem efeito protetor na incidência de diarréia e de infecção respiratória 26 , nem na mortalidade por doença respiratória aguda 27 .

Em relação ao impacto da suplementação em recém-nascidos pré-termo (até 32 semanas), Darlow \& Graham 28 não relatam redução na mortalidade, na duração do uso de oxigênio, na ocorrência de retinopatia ou de septicemia. Porém, há impacto da intervenção quando o uso de oxigênio e a ocorrência de morte são considerados conjuntamente na análise agregada. Houve também efeito protetor em relação ao uso de oxigênio em recém-nascidos antes de 36 semanas de idade gestacional corrigida.

Ramakhrishnan et al. 29 não referem efeito benéfico da suplementação de vitamina A no crescimento e desenvolvimento físico de crianças e adolescentes. Irlam et al. 30 e Wiysonge et al. ${ }^{31}$, que revisaram o efeito da suplementação em crianças, adultos e gestantes com HIV, relatam efeito protetor somente no grupo infantil, com redução na mortalidade geral e por causas relacionadas à AIDS, redução na morbidade por diarréia e aumento de peso ao nascer.

\section{Discussão}

Tanto a deficiência de vitamina A quanto a morbidade e a mortalidade associadas carac- terizam-se como um importante problema de saúde pública. Há evidências de que a suplementação de vitamina A em crianças de regiões endêmicas de deficiência dessa vitamina seja protetora em relação à mortalidade geral, tendo em vista os resultados semelhantes de três metanálises conduzidas por Fawzi et al. 20, Glasziou \& Mackerras 21 e Beaton et al. 22, que descrevem redução de $23 \%$ a $30 \%$ no risco de morte. O efeito protetor em relação à mortalidade é evidente em crianças que apresentam quadro de diarréia e de sarampo 20,21,23; recentemente, estudos também têm apontado para um papel protetor da suplementação de vitamina $\mathrm{A}$ em relação à mortalidade em crianças com AIDS 30.

Apesar do evidente impacto da vitamina A na atenuação do quadro de diarréia e sarampo, metanálises que investigaram efeito protetor em relação à incidência de diarréia e de infecções respiratórias 26 , à incidência de peumonia e mortalidade por essa doença 24 e de doença respiratória aguda 27 não descrevem resultados positivos.

Segundo Ni et al. 25 e Brown \& Roberts 27, a ausência de evidência significativa na redução da mortalidade, da morbidade e no curso clínico da doença em crianças com pneumonia ou com doença respiratória aguda pode ser explicada pelo limitado número de estudos incluídos na análise e conseqüente diminuição de poder estatístico para detectar diferenças significativas. Deve-se considerar também que outros fatores, como vieses de publicação, heterogeneidade entre os estudos incluídos nas metanálises (diferentes populações, diferenças metodológicas e outras), diferenças na fisiopatologia dessas doenças, podem estar relacionados com a ausência de impacto. 
Grotto et al. 26 apontam que distribuição de altas doses de vitamina A a pré-escolares tem pouco valor na prevenção primária de doenças diarréicas ou infecções agudas do trato respiratório, podendo até causar uma elevação na incidência de infecções sintomáticas do trato respiratório. Por essa razão, os autores reforçam a recomendação da suplementação somente em áreas de deficiência de vitamina $\mathrm{A}$.

Em relação às metanálises que investigaram o impacto, no sarampo, da utilização de vitamina A, recomendou-se, em uma primeira metanálise, a administração de altas doses dessa vitamina para crianças com a doença 20. Em metanálise mais recente 23 , apóia-se a recomendação da Organização Mundial da Saúde (OMS) de que sejam administradas duas doses de 200.000UI de vitamina A em crianças abaixo de dois anos com quadro grave de sarampo, em adição ao tratamento convencional. A evidência dos estudos só pode ser generalizada aos países em desenvolvimento, pois, na realidade, dispõe-se de somente uma metanálise realizada em país desenvolvido (Japão), na qual se utilizou uma dose reduzida de vitamina A (100.000UI).

Para o desfecho mortalidade infantil, observa-se em extensa revisão ${ }^{28}$ que a suplementação de vitamina A em recém-nascidos com muito baixo peso (peso $<1.500 \mathrm{~g}$ ) somente está associada a benefícios quando este fator é considerado conjuntamente com a mortalidade ou com as necessidades de oxigênio com um mês de vida. A maior parte dos estudos, porém, não avalia qual é a melhor via nem qual é a melhor dose para suplementação, embora aparentemente os recém-nascidos com concentração sérica de retinol abaixo de 0,70 $\mu \mathrm{mol} / \mathrm{L}$ (deficiência de vitamina A) respondam positivamente a megadose de vitamina A (100.000UI ou 200.000UI). O maior benefício resulta de dois estudos envolvendo recém-nascidos com extremo baixo peso ao nascimento $(<1.000 \mathrm{~g}) 32,33$.

Nas metanálises que avaliam o impacto exercido pela administração de vitamina A durante a gravidez e lactação sobre as alterações clínicas e laboratoriais maternas e dos recém-nascidos, observou-se em dois estudos, do Nepal 11 e da Indonésia ${ }^{14}$, um efeito benéfico. No entanto, os autores concluem que é necessária a condução de estudos subseqüentes para se afirmar que a suplementação de vitamina A realmente tenha influência na redução da mortalidade e da morbidade maternas, questionando-se, inclusive, o mecanismo pelo qual esta possa ocorrer ${ }^{9}$. No estudo do Nepal, as informações sobre o óbito materno foram obtidas por meio de entrevista com um familiar (autópsia verbal), que é metodologia útil em investigações em populações, estando, porém, sujeita a imprecisão e erros de classificação. Outro ponto importante em relação à mortalidade foi o fato de haver descrição de impacto somente para a mortalidade geral (incluindo morte por doenças crônicas e causas externas); há ausência de efeito quando se consideraram somente as causas obstétricas.

Embora as intervenções com vitamina A ou ferro, isoladamente, produzam benefícios, como elevação nas concentrações de hemoglobina e redução da mortalidade, elas não mostram impacto importante no crescimento linear de crianças, ao contrário de intervenções que utilizam vários micronutrientes e/ou zinco, que apresentam excelente efeito ${ }^{29}$. Sendo assim, a interação entre os micronutrientes deve ser considerada no planejamento de programas de saúde pública, pois, em geral, múltiplas deficiências nutricionais coexistem em populações de áreas menos desenvolvidas. Há, até mesmo, estudos que sugerem que a suplementação combinada de zinco e vitamina A seja mais efetiva na atenuação do quadro de diarréia do que a utilização de somente vitamina A 34 .

No entanto, é importante lembrar que, em regiões mais carentes, a suplementação pode ser um sério problema em termos de implementação, optando-se pela fortificação da dieta, melhora da qualidade desta e educação, na tentativa de se obter melhor ingestão de micronutrientes por crianças 29 .

Apesar de um dos estudos incluídos na presente revisão analisar o impacto da suplementação em crianças com extremo baixo peso ao nascer, não se diferenciam crianças pré-termo de crianças com restrição de crescimento intrauterino. No entanto, estudo desenvolvido no Brasil, na década de 90 35, mostra que os níveis de vitamina A são maiores em recém-nascidos com peso adequado para a idade gestacional do que em recém-nascidos com restrição de crescimento intra-uterino, independentemente dos níveis maternos de vitamina A. Baseando-se nesse estudo inédito na literatura internacional, fazem-se necessários estudos que avaliem o impacto da suplementação em lactantes que geram recém-nascidos com peso adequado para a idade gestacional e recém-nascidos com restrição de crescimento intra-uterino.

\section{Conclusões}

Há evidências de que a suplementação de vitamina A em crianças esteja associada com redução em torno de $23 \%$ a $30 \%$ na mortalidade geral de crianças com idade entre seis meses a cinco anos. Sugere-se, também, que a intervenção ate- 
nue a gravidade dos quadros de diarréia e sarampo, refletindo na redução do risco de morte associado a essas doenças.

Não há evidências de que a suplementação de vitamina A em crianças desempenhe papel protetor em relação à incidência de pneumonia não associada ao sarampo e mortalidade por essa causa.

Estudos mais recentes indicam que a vitamina A desempenha papel protetor em relação ao peso ao nascer e à redução da mortalidade em crianças que vivem com AIDS.

Não há evidência de que a suplementação direcionada às gestantes e lactantes esteja associada à redução da morbidade e da mortalidade infantis. Há indicação de que essa intervenção seja protetora em relação à morbidade materna, mas há necessidade de estudos subseqüentes que avaliem o impacto na mortalidade.

\section{Resumo}

O objetivo deste artigo é reunir os resultados de revisões sistemáticas e metanálises sobre o efeito da suplementação de vitamina A no crescimento, morbimortalidade infantil, materna e fetal. Foi realizada uma busca criteriosa nas bases de dados bibliográficos PubMed, Embase, LILACS, PAHO, Biblioteca Cochrane, Banco de Teses da CAPES, Biblioteca Digital de Teses da USP e acervo da Biblioteca Central da UNIFESP, localizando-se 14 trabalhos publicados entre $1993 \mathrm{e}$ 2006. Há evidências de que a suplementação de vitamina A em crianças esteja associada à redução de $23 \%$ a $30 \%$ no risco de morte e atenuação da gravidade do quadro de sarampo e diarréia. Não há evidências de que a intervenção em crianças reduza a incidência de pneumonia não associada ao sarampo e mortalidade por essa causa. Em crianças e gestantes com HIVIAIDS, a suplementação apresenta impacto positivo na morbi-mortalidade infantil e no peso ao nascer. Não há evidências de que a suplementação em gestantes e lactantes esteja associada à redução da morbi-mortalidade infantil, mas há indicação de que essa intervenção seja protetora em relação à morbidade materna.

Vitamina A; Deficiência de Vitamina A; Saúde Materno-Infantil; Metanálise [Tipo de Publicação]

\section{Colaboradores}

J. M. Oliveira realizou a identificação dos estudos, extração dos resultados e redação do artigo. P. H. C. Rondó participou na redação do artigo. 


\section{Referências}

1. Tomkins A. Malnutrition, morbidity and mortality in children and their mothers. Proc Nutr Soc 2000; 59:135-46.

2. West Jr. KP, Rice A, Sugimoto J. Tables on the global burden of vitamin A deficiency and xerophthalmia among preschool aged children and low vitamin A status, vitamin A deficiency and night blindness among pregnant women by WHO region. http:// www.jhsph.edu/CHN/GlobalVAD.pdf (acessado em 25/Out/2006).

3. Green HN, Mellamby E. Vitamin A as an anti-infective agent. BMJ 1928; 3:691-6.

4. Elisson JB. Intensive vitamin therapy in measle. BMJ 1932; 2:708-11.

5. Sommer A, Trawotjo I, Djunacdi E, West Jr. KP, Loeden AA, Tiden R, et al. Impact of vitamin A supplementation on childhood mortality. A randomized controlled trial. Lancet 1986; 1:1169-73.

6. World Health Organization. Safe vitamin A dosage during pregnancy and lactation: recommendations and report of a consultation. Geneva: World Health Organization; 1998.

7. World Health Organization. Vitamin A supplements: a guide to their use in treatment and prevention of vitamin A deficiency and xerophthalmia. 2nd Ed. Geneva: World Health Organization/ United Nations Children's Fund/International Vitamin A Consultative Group Task Force; 1997.

8. Joint United Nations Programme on AIDS. AIDS in Africa: three scenarios to 2025. Geneva: Joint United Nations Programme on AIDS; 2005.

9. van den Broek N, Kulier R, Gulmezoglu AM, Villar J. Vitamin A supplementation during pregnancy. Cochrane Database Syst Rev 2002; (4):CD001996.

10. Oliveira JM. Suplementação de vitamina A em lactantes: revisão sistemática [Dissertação de Mestrado]. São Paulo: Faculdade de Saúde Pública, Universidade de São Paulo; 2006.

11. West Jr. KP, Katz J, Khatry SK, Leclerq SC, Pradhan EK, Shrestha SR, et al. Double blind, cluster randomised trial of low dose supplementation with vitamin A or beta carotene on mortality related to pregnancy in Nepal. BMJ 1999; 318:570-5.

12. Christian P, West Jr. KP, Khatry SK, Katz J, Leclerq SC, Kimbrough-Pradhan E, et al. Vitamin A or beta-carotene supplementation reduces symptoms of illness in pregnant and lactating Nepali women. J Nutr 2000; 130:2675-82.

13. Katz J, West Jr. KP, Khatry SK, Pradhan EK, Leclerq SC, Christian P, et al. Maternal low-dose vitamin A or beta-carotene supplementation has no effect on fetal loss and early infant mortality: a randomized cluster trial in Nepal. Am J Clin Nutr 2000; 71:15706.

14. Suharno D, West CE, Muhilal, Karyadi D, Hautvast JG. Supplementation with vitamin A and iron for nutritional anaemia in pregnant women in West Java, Indonesia. Lancet 1993; 342:1235-8.

15. Semba RD, Kumwenda N, Taha TE, Mtimavalye L, Broadhead R, Garret E, et al. Impact of vitamin A supplementation on anaemia and plasma erythropoietin concentrations in pregnant women: a controlled clinical trial. Eur J Haematol 2001; 66:38995.
16. van den Broek NR, White SA, Flowers C, Cook JD, Letsky EA, Tanumihardjo SA, et al. Randomised trial of vitamin A supplementation in pregnant women in rural Malawi found to be anaemic on screening by HemoCue. BJOG 2006; 113:569-76.

17. Randomised trial to assess benefits and safety of vitamin A supplementation linked to immunisation in early infancy. WHO/CHD ImmunisationLinked Vitamin A Supplementation Study Group. Lancet 1998; 352:1257-63.

18. Malaba LC, Iliff PJ, Nathoo KJ, Marinda E, Moulton LH, Zijenah LS, et al. Effect of postpartum maternal or neonatal vitamin A supplementation on infant mortality among infants born to HIV-negative mothers in Zimbabwe. Am J Clin Nutr 2005; 81:454-60

19. Venkatarao T, Ramakrishnan R, Nair NG, Radhakrishnan S, Sundaramoorthy L, Koya PK, et al. Effect of vitamin A supplementation to mother and infant on morbidity in infancy. Indian Pediatr 1996; 33:279-86.

20. Fawzi WW, Chalmers TC, Herrera MG, Mosteller F Vitamin A supplementation and child mortality: a meta-analysis. JAMA 1993; 269:898-903.

21. Glasziou PP, Mackerras DE. Vitamin A supplementation in infectious diseases: a meta-analysis. BMJ 1993; 306:366-70.

22. Beaton GH, Martorell R, Aronson KA, Edmonston B, McCabe G, Ross AC, et al. La suplementación con vitamina A y la morbilidad y mortalidad infantil en los países en desarrollo. Bol Oficina Sanit Panam 1994; 117:506-18.

23. Huiming Y, Chaomin W, Meng M. Vitamin A for treating measles in children. Cochrane Database Syst Rev 2005; (4):CD001479.

24. Potential interventions for the prevention of childhood pneumonia in developing countries: a metaanalysis of data from field trials to assess the impact of vitamin A supplementation on pneumonia morbidity and mortality. The Vitamin A and Pneumonia Working Group. Bull World Health Organ 1995; 73:609-19.

25. Ni J, Wei J, Wu T. Vitamin A for non-measles pneumonia in children. Cochrane Database Syst Rev 2005; (3):CD003700.

26. Grotto I, Mimouni M, Gdalevich M, Mimouni D. Vitamin A supplementation and childhood morbidity from diarrhea and respiratory infections: a meta-analysis. J Pediatr 2003; 142:297-304.

27. Brown N, Roberts C. Vitamin A for acute respiratory infection in developing countries: a meta-analysis. Acta Paediatr 2004; 93:1437-42.

28. Darlow BA, Graham PJ. Vitamin A supplementation for preventing morbidity and mortality in very low birthweight infants. Cochrane Database Syst Rev 2002; (4):CD000501.

29. Ramakrishnan U, Aburto N, McCabe G, Martorell R. Multimicronutrient interventions but not vitamin a or iron interventions alone improve child growth: results of 3 meta-analyses. J Nutr 2004; 134:2592-602. 
30. Irlam JH, Visser ME, Rollins N, Siegfried N. Micronutrient supplementation in children and adults with HIV infection. Cochrane Database Syst Rev 2005; (4):CD003650.

31. Wiysonge CS, Shey MS, Sterne JA, Brocklehurst P. Vitamin A supplementation for reducing the risk of mother-to-child transmission of HIV infection. Cochrane Database Syst Rev 2005; (4):CD003648.

32. Tyson JE, Wright LL, Oh W, Kennedy KA, Mele L, Ehrenkranz RA, et al. Vitamin A supplementation for extremely-low-birth-weight infants. N Engl J Med 1999; 340:1962-8.
33. Wardle SP, Hughes A, Chen S, Shaw NJ. Randomised controlled trial of oral vitamin A supplementation in preterm infants to prevent chronic lung disease. Arch Dis Child Fetal Neonatal Ed 2001; 84:F9-13.

34. Rahman MM, Vermund SH, Wahed MA, Fuchs GJ Baqui AH, Alvarez JO. Simultaneous zinc and vitamin A supplementation in Bangladeshi children: randomised double blind controlled trial. BM] $2001 ; 323: 314-8$.

35. Rondó PH, Abbott R, Tomkins AM. Vitamin A and intrauterine growth retardation. J Pediatr (Rio de J) 1997; 73:335-9.

36. Association of Official Analytical Chemists. Official methods of analysis. 14th Ed. Arlington: Association of Official Analytical Chemists; 1984.

Recebido em 13/Nov/2006

Versão final reapresentada em 07/Mai/2007

Aprovado em 16/Mai/2007 